\title{
Editor's Preface for the Special Issue Dedicated to the 2015 JETC
}

\author{
Jean-Noël Jaubert ${ }^{1}$ • Romain Privat ${ }^{1}$. \\ Michel Feidt ${ }^{2}$
}

The 13th Joint European Thermodynamics Conference (JETC) was held at Lorraine University in Nancy (France) from May 20 to May 22, 2015.

This conference is now well established with a periodicity of two years. Previous conferences took place successively in Compiègne, Brussels, Lausanne, Nancy, Marseille, Toulouse, Mons, Barcelona, Saint-Etienne, Copenhagen, Chemnitz, and Brescia. JETC is European based but welcomes authors from all over the world, as can be seen from the list of about 270 contributors to this 13th conference. It is on purpose a small size meeting, often taking place in academic premises and with limited registration fee.

JETC is traditionally built on the heritage of Ilya Prigogine, who personally participated in some of the first meetings. In the framework of the conference, this is why the Ilya Prigogine Prize of Thermodynamics is being conferred since 2001 to a young scientist for his PhD thesis. This year's laureate, selected by an international committee, was Ronan McGovern from Ireland, who defended his thesis at MIT, on the thermodynamics of future membrane desalination processes.

Although all domains of Thermodynamic Science are welcome, the scientific flavor of JETC, influenced by Prigogine's ideas strongly focuses on non-equilibrium thermodynamics, with topics related to physics, chemistry, biology, engineering, mathematics, and even cosmology. The approaches range from phenomenological

This article is part of the 13th Joint European Thermodynamics Conference Special Issue.

$\triangle \quad$ Jean-Noël Jaubert

Jean-Noel.Jaubert@univ-lorraine.fr

1 Université de Lorraine, École Nationale Supérieure des Industries Chimiques, Laboratoire Réactions et Génie des Procédés - UMR CNRS 7274, 1 rue Grandville, 54000 Nancy, France

2 Université de Lorraine, Laboratoire d'Energétique et de Mécanique Théorique et Appliquée - UMR CNRS 7563, 2 Avenue de la Forêt de Haye, 54504 Vandoeuvre les Nancy, France 
thermodynamics to statistical, molecular, and quantum thermodynamics. The papers selected for this special issue reflect all these aspects.

The next JETC conference will take place in Budapest in 2017.

The editors wish to thank the authors who have submitted papers to this topical issue and the reviewers who have taken time to review and comment on the submissions. 